Pacific Journal of Mathematics

ENDOMORPHISMS OF RANK ONE MIXED MODULES OVER DISC RTE VALUE RINGS 


\title{
ENDOMORPHISMS OF RANK ONE MIXED MODULES OVER DISCRETE VALUATION RINGS
}

\author{
WARREN MAY AND ElIAS TOUBASSI
}

\begin{abstract}
Mixed modules over a discrete valuation ring with torsion-free rank one and simply presented torsion submodules are considered. It is proved that every isomorphism of endomorphism algebras of such modules is induced by an isomorphism of the modules.
\end{abstract}

We begin by recalling some relevant results. A simple antecedent of the Noether-Skolem theorem states that if $V$ is a vector space over a field $F$, then every automorphism of the endomorphism algebra of $V$ is inner. $\mathrm{R}$. Baer proved a similar result for bounded $p$-primary groups which was subsequently generalized by Kaplansky to torsion modules over discrete valuation rings. Fix a discrete valuation ring $R$ (and choose a prime element $p$ ). If $M$ is an $R$-module, we denote the endomorphism algebra of $M$ by $E(M)$. In this paper, we shall prove the following

THEOREM. Let $M$ be an $R$-module of torsion-free rank one with simply presented torsion submodule. If $N$ is an $R$-module of torsion-free rank one, then every algebra isomorphism $\Phi: E(M) \rightarrow E(N)$ is induced by an isomorphism $\theta: M \rightarrow N$ such that $\Phi(\alpha)=\theta \alpha \theta^{-1}$ for every $\alpha \in E(M)$.

COROLlaRY. Every algebra automorphism of $E(M)$ is inner.

We remark that the isomorphism $\theta$ is not obtained by applying the familiar invariants for rank one modules with simply presented torsion, but is constructed directly. We have restricted our attention to modules over discrete valuation rings in view of an example in [5] which suggests that the theorem does not generalize to modules over principal ideal domains which have infinitely many primes.

To fix notation, let $K$ denote the quotient field of $R$. Moreover, we shall always let $M$ and $N$ denote $R$-modules of torsion-free rank one, with respective torsion submodules $T(M)$ and $T(N)$. If $\Phi: E(M) \rightarrow E(N)$ is an $R$-algebra isomorphism, then there exists an isomorphism

$$
\phi: T(M) \rightarrow T(N)
$$


such that $\left.\Phi(\alpha)\right|_{T(N)}=\phi \alpha \phi^{-1}$ for every $\alpha \in E(M)$. This is a consequence of Kaplansky's theorem [2, Theorem 28] and the following observation. If $\varepsilon \in E(M)$ is an idempotent such that $\varepsilon(M) \cong K / R$, then $\Phi(\varepsilon)(N)$ cannot be torsion-free since $\operatorname{Hom}((1-\varepsilon)(M), \varepsilon(M)) \neq 0$; hence $\Phi(\varepsilon)(N) \cong$ $K / R$. Throughout the paper, we shall usually denote the endomorphism $\Phi(\alpha)$ by $\alpha^{*}$.

The proof of the theorem will be in two cases, depending on whether $M$ is split or nonsplit. First we dispose of the split case.

LEMMA 1. Let $\Phi: E(M) \rightarrow E(N)$ be an R-algebra isomorphism, and assume that $M$ is a split module. Then there exists an isomorphism $\theta$ : $M \rightarrow N$ such that $\Phi(\alpha)=\theta \alpha \theta^{-1}$ for every $\alpha \in E(M)$.

Proof. We may write $M=F \oplus T$, where $T=T(M)$ and $F$ is isomorphic to either $R$ or $K$. Let $\varepsilon \in E(M)$ be an idempotent such that $\varepsilon(M)=F$ and $\varepsilon(T)=0$. Then $\varepsilon^{*}$ yields a decomposition $N=F^{\prime} \oplus T^{\prime}$, where $\varepsilon^{*}(N)=F^{\prime}$ and $\varepsilon^{*}\left(T^{\prime}\right)=0$. Since $E\left(F^{\prime}\right) \cong E(F) \cong R$ or $K$, we may conclude from the observation following (A) that $F^{\prime} \cong F$ and that $T^{\prime}=T(N)$.

First suppose that $F \cong R$. Then we may choose generators $f \in F$ and $f^{\prime} \in F^{\prime}$, which in turn determine isomorphisms $E(M) \varepsilon \cong M$ and $E(N) \varepsilon^{*}$ $\cong N$ by evaluation. Since $\Phi$ takes $E(M) \varepsilon$ to $E(N) \varepsilon^{*}$, by composition we obtain an isomorphism $\theta: M \rightarrow N$. Let $\alpha \in E(M)$. Any element of $M$ may be represented as $\beta(f)$ for some $\beta \in E(M) \varepsilon$. Then $\theta \alpha(\beta(f))=$ $(\alpha \beta)^{*}\left(f^{\prime}\right)=\alpha^{*} \theta(\beta(f))$. Thus $\theta \alpha=\alpha^{*} \theta$, and we are done.

Now suppose that $F \cong K$. Let $D$ and $D^{\prime}$ denote the maximal divisible submodules of $T$ and $T^{\prime}$ respectively. If $D \neq 0$, choose a nonzero map $\eta \in E(M)$ with $\eta(F) \subseteq T, \eta(T)=0$, and choose a generator $f$ of the kernel of $\eta$ on $F$. If $D=0$, take $\eta=0$ and choose $f$ to be any nonzero element of $F$. We have a homomorphism $E(M) \varepsilon \rightarrow F \oplus D$ given by evaluation at $f$. This homomorphism is surjective, and since $\eta(M)$ is a summand of $M$, the kernel can be expressed as $E(M) \eta$. But $F^{\prime} \cong K$, $\eta^{*}\left(F^{\prime}\right) \subseteq T^{\prime}$ and $\eta^{*}\left(T^{\prime}\right)=0$. Thus, in a similar fashion, we may choose a generator $f^{\prime}$ of the kernel of $\eta^{*}$ on $F^{\prime}$ (or any nonzero element of $F^{\prime}$ in case $\eta^{*}=0$ ). By evaluation at $f^{\prime}$, we obtain a surjective homomorphism $E(N) \varepsilon^{*} \rightarrow F^{\prime} \oplus D^{\prime}$ with kernel $E(N) \eta^{*}$. Since $\Phi$ takes $E(M) \eta$ to $E(N) \eta^{*}$, we obtain, as above, an isomorphism $\theta_{0}: F \oplus D \rightarrow F^{\prime} \oplus D^{\prime}$ such that $\theta_{0} \alpha(x)=\alpha^{*} \theta_{0}(x)$ for every $\alpha \in E(M)$ and $x \in F \oplus D$. By (A), we may choose an isomorphism $\phi: T \rightarrow T^{\prime}$ such that $\phi \alpha(x)=\alpha^{*} \phi(x)$ for every 
$\alpha \in E(M)$ and $x \in T$. Since $\left.\alpha\right|_{D}$ ranges over $E(D)$, we see that $\left.\phi^{-1} \theta_{0}\right|_{D}$ lies in the center of $E(D)$. Thus it is given by the action of a unit in the completion of $R$. Alter $\phi$ by this unit factor. Then $\left.\phi\right|_{D}=\left.\theta_{0}\right|_{D}$ and $\phi \alpha=\alpha^{*} \phi$ still holds. Consequently, $\theta=\theta_{0} \cup \phi$ is an isomorphism of $M$ to $N$ such that $\theta \alpha=\alpha^{*} \theta$ for every $\alpha \in E(M)$.

The nonsplit case will be more substantial. Let $x$ be a torsion-free element of $M$. We call $x$ principal if $\langle x\rangle$ is the kernel of a family of maps in $E(M)$ (i.e. the common kernel of the individual maps in the family). We denote the $p$-length of a torsion module $T$ by $l(T)$, except that we put $l(T)=\infty$ if $T$ is not reduced.

Lemma 2. Assume $T(M)$ is simply presented and that $x \in M$ is a torsion-free element. Then the following are equivalent:

(1) $x$ is principal;

(2) $\langle x\rangle$ is the kernel of the family of endomorphisms vanishing on $x$;

(3) $l(M /\langle x\rangle)=l(T(M))$, where l denotes p-length;

(4) for every $v_{1}, v_{2} \in M$ with $v_{1} \notin\left\langle v_{2}, x\right\rangle$, there exists $\alpha \in E(M)$ with $\alpha\left(v_{1}\right) \neq 0, \alpha\left(\left\langle v_{2}, x\right\rangle\right)=0$.

Proof. It is evident that (1) implies (2). Endomorphisms vanishing on $x$ map into $T(M)$, thus $l(T(M))<l(M /\langle x\rangle)$ contradicts (2). But clearly, $l(T(M)) \leq l(M /\langle x\rangle)$, therefore (2) implies (3). Now assume (3), and suppose that $v_{1}$ and $v_{2}$ are as given in (4). Let $\bar{M}=M /\langle x\rangle$. Then there exists a map $\bar{\gamma}:\left\langle\bar{v}_{1}, \bar{v}_{2}\right\rangle \rightarrow T(M)$ such that $\bar{\gamma}\left(\bar{v}_{1}\right) \neq 0, \bar{\gamma}\left(\bar{v}_{2}\right)=0$, and $\bar{\gamma}$ does not decrease $p$-heights taken in $\bar{M}$. By applying [6, Theorem 1] and [1, Corollary 81.4], suitably extended to $R$-modules, we see that $\bar{M}$ is simply presented, $\left\langle\bar{v}_{1}, \bar{v}_{2}\right\rangle$ is a nice submodule, and $\bar{\gamma}$ can be extended to a homomorphism on $\bar{M}$. This induces the homomorphism $\alpha$ that demonstrates (4). Finally, it is clear that (4) implies (1).

The principal elements are torsion-free elements which are accessible via the endomorphism ring. In the proof of the nonsplit case, we shall construct an isomorphism $\theta$ by sending principal elements to corresponding principal elements. Before making this correspondence precise, we need a brief lemma on the Ulm invariants of $M /\langle x\rangle$. If $Q$ is an $R$-module, $u \in Q$, and $\rho$ an ordinal, then $f_{\rho}(Q)$ will denote the $\rho$ th Ulm invariant of $Q$, and $h(u)$ will denote the $p$-height of $u$. We say that the $p$-indicator of $u$ has a gap at $h\left(p^{r} u\right)$ if $h\left(p^{r+1} u\right)>h\left(p^{r} u\right)+1$. 
Lemma 3. Let $x \in M$ be torsion-free, $\bar{M}=M /\langle x\rangle$, and $T=T(M)$. Let $\rho$ be an ordinal. Then $f_{\rho}(\bar{M})=f_{\rho}(T)$ except in the following cases:

(1) if the p-indicator of $x$ has a gap at $h\left(p^{r} x\right)$ and $h\left(p^{r} x\right)=\rho$, then $f_{\rho}(\bar{M})+1=f_{\rho}(T)$

(2) if either the p-indicator of $x$ has a gap at $h\left(p^{r} x\right)$ and $h\left(p^{r+1} x\right)=\rho$ +1 , or else $h(x)=\rho+1$, then $f_{\rho}(\bar{M})=f_{\rho}(T)+1$.

Proof. Let $T_{\rho}=p^{\rho} T[p]$ and $S_{\rho}=p^{\rho} \bar{M}[p]$. We shall regard $T$ as naturally embedded in $\bar{M}$, thus $T_{\rho} \subseteq S_{\rho}$. It suffices to prove that $T_{\rho}=S_{\rho}$ except when either $\rho<h(x)$ or $h\left(p^{k-1} x\right)<\rho<h\left(p^{k} x\right)$ for some $k>0$, in which case $S_{\rho} / T_{\rho}$ has dimension one over $R /\langle p\rangle$. First suppose that either $\rho<h(x)$ or $h\left(p^{k-1} x\right)<\rho<h\left(p^{k} x\right)$. We may choose $v \in M$ such that $p v=p^{k} x(k \geq 0)$, and $h(v) \geq \rho$. Thus, $\bar{v} \in S_{\rho}$. If $k=0$, then $\bar{v} \notin T$; if $k>0$, then $h\left(v-p^{k-1} x\right)<\rho$ implies that $\bar{v} \notin T_{\rho}$. Consequently, $S_{\rho} \neq$ $T_{\rho}$. Now suppose that $w \in M$ satisfies $\bar{w} \in S_{\rho}, \bar{w} \notin T_{\rho}$. Since $\langle x\rangle$ is nice (see [1, Lemma 104.1]), we may assume that $h(w)=h(\bar{w}) \geq \rho$. Thus $w$ is torsion-free, and we have $p w=p^{m} a x(a \in R, p \nmid a)$. Therefore $\rho<$ $h\left(p^{m} x\right)$, and if $m>0$, then $h\left(p^{m-1} x\right)<\rho$ since $w-p^{m-1} a x \notin T_{\rho}$. Hence, either $\rho<h(x)$ or $h\left(p^{k-1} x\right)<\rho<h\left(p^{k} x\right)$, where $k=m$. If $v$ is taken as above, then $w-a v \in T_{\rho}$, hence the dimension of $S_{\rho} / T_{\rho}$ is one.

We now make the correspondence of principal elements precise. Let $x \in M$ be torsion-free. Define $I(M)=\{\alpha \in E(M) \mid \alpha(M) \subseteq T(M)\}$ and $I(M, x)=\{\alpha \in I(M) \mid \alpha(x)=0\}$.

LeMMA 4. Let $\Phi: E(M) \rightarrow E(N)$ be an R-algebra isomorphism. Assume $M$ is non-split and that $T(M)$ is simply presented. If $x$ is a principal element of $M$, then there exists a principal element $y$ of $N$ such that $\Phi(I(M, x))=I(N, y)$. Moreover, $y$ is unique up to a unit multiple.

Proof. Choose an isomorphism by (A) to identify $T(M)$ with $T(N)$. Let us use $T$ to denote this common submodule. The proof will consist of two parts.

(1) First we shall show that $\Phi(I(M))=I(N)$. Let $\alpha \in I(M)$. Our hypothesis is symmetric by Lemma 1 , hence it suffices to show that $\alpha^{*} \in I(N)$. Suppose that $T$ is not reduced. If $\alpha^{*}(N) \nsubseteq T$, then there exists $\delta \in E(M)$ such that $\delta^{*}(T)=0$ and $\delta^{*} \alpha^{*} \neq 0$. But $\delta(T)=0$, hence $\delta \alpha=0$. This contradiction implies that $\alpha^{*} \in I(N)$ in this case, hence, we may assume that $T$ is reduced. Let $l(T)=\sigma+n(\sigma$ limit ordinal, $n<\omega)$. We claim that there exists $\Delta \in I(M)$ such that the kernel of $\Delta$ is 
$\left\langle x, p^{\sigma} T\right\rangle$, the cokernel of $\Delta$ is reduced, and $\Delta^{*} \in I(N)$. Since $T$ is simply presented, we may decompose it as $T=T_{1} \oplus T_{2}$, where $\Sigma_{\tau \leq \rho} f_{\rho}\left(T_{i}\right)=$ $\Sigma_{\tau \leq \rho} f_{\rho}(T)$ for every $\tau<\sigma, i=1,2$. It follows from Lemma 3 that $\sum_{\tau \leq \rho} f_{\rho}\left(M /\left\langle x, p^{\sigma} T\right\rangle\right)=\Sigma_{\tau \leq \rho} f_{\rho}\left(T_{i}\right)$, hence the main result in [4], generalized to modules over $R$, implies that there exist $\beta_{i}: M \rightarrow T_{i}$ such that the kernel of $\beta_{i}$ is $\left\langle x, p^{\sigma} T\right\rangle$ and the cokernel of $\beta_{i}$ is reduced $(i=1,2)$. By interchanging indices if necessary, there exists $r \in R$ such that $\beta_{1}^{*}-r \beta_{2}^{*}$ $\in I(N)$. Put $\Delta=\beta_{1}-r \beta_{2}$. It is easy to see that $\Delta$ meets the requirements of the claim. We may now choose $m>0$ such that $\left\langle x, p^{\sigma} T\right\rangle$ is contained in the kernel of $p^{m} \alpha$. Thus there exists $\gamma: \Delta(M) \rightarrow T$ such that $p^{m} \alpha=\gamma \Delta$. Since $M$ is nonsplit, we must have $\Delta(M) / \Delta(T) \cong K / R$. But $T / \Delta(M)$ is reduced, hence $\Delta(M) / \Delta(T)$ is the maximal divisible submodule of $T / \Delta(T)$. Similar statements apply to $N$ and $\Delta^{*}(N)$, hence we may conclude that $\Delta(M)=\Delta^{*}(N)$ since $\Delta(T)=\Delta^{*}(T)$. It follows that the composition $\gamma \Delta^{*}$ makes sense, and $\gamma \Delta^{*} \in I(N)$. But $(\gamma \Delta)^{*}$ and $\gamma \Delta^{*}$ agree on $T$, hence they are equal since $T$ is reduced and $N / T$ is divisible. Thus $p^{m} \alpha^{*}=(\gamma \Delta)^{*}=\gamma \Delta^{*} \in I(N)$; consequently $\alpha^{*} \in I(N)$.

(2) Decompose $M$ as $M=M_{0} \oplus D$, where $D$ is the maximal divisible submodule of $T$. Then $T=T_{0} \oplus D$, where $T_{0}=T\left(M_{0}\right)$. Note that (1) implies that $\Phi$ takes $\operatorname{Hom}\left(M, T_{0}\right)$ to $\operatorname{Hom}\left(N, T_{0}\right)$. As in (1), we see that

$$
\sum_{\tau \leq \rho} f_{\rho}(M /\langle x\rangle) \leq \sum_{\tau \leq \rho} f_{\rho}\left(T \oplus T_{0}\right)
$$

hence by [4] (and an isomorphism on $D$ ) there exists a homomorphism $\Delta$ : $M \rightarrow T \oplus T_{0}$ with kernel $\langle x\rangle$ and reduced cokernel. We may represent $\Delta$ as the product map $\Delta=\left(\beta_{1}, \beta_{2}\right)$, where $\beta_{1} \in I(M)$ and $\beta_{2} \in$ $\operatorname{Hom}\left(M, T_{0}\right)$. Define $\Delta^{\prime}: N \rightarrow T \oplus T_{0}$ by $\Delta^{\prime}=\left(\beta_{1}^{*}, \beta_{2}^{*}\right)$. Since $\Delta$ is injective on $T$, it follows that $\Delta^{\prime}$ is injective on $T$. Since $N$ is nonsplit, the kernel of $\Delta^{\prime}$ is generated by a torsion-free element $y \in N$. Clearly $y$ is principal since it is the kernel of $\left\{\beta_{1}^{*}, \beta_{2}^{*}\right\}$. We must now show that $\Phi(I(M, x))=I(N, y)$. If this is so, then $y$ will be unique up to a unit multiple since if $z$ is another element with the same properties, then $z$ principal and $I(N, y)=I(N, z)$ imply that $\langle y\rangle=\langle z\rangle$.

By arguments similar to those in (1) with $\Delta^{\prime}$ replacing $\Delta^{*}$, we see that $\Delta(M)=\Delta^{\prime}(N)$. Now let $\alpha \in I(M, x)$. It suffices to consider cases $\alpha(M)$ $\subseteq T_{0}$ and $\alpha(M) \subseteq D$, since any element in $I(M, x)$ is the sum of two such maps. First suppose $\alpha(M) \subseteq T_{0}$. There exists a map $\gamma: \Delta(M) \rightarrow T_{0}$ such that $\alpha=\gamma \Delta$. Again, as in (1), we have $\alpha^{*}=(\gamma \Delta)^{*}=\gamma \Delta^{\prime} \in I(N, y)$. Now suppose that $\alpha(M) \subseteq D$. Since $D$ is divisible and $\Delta(M) \subseteq M \oplus M$, there exists a map $\gamma: M \oplus M \rightarrow D$ such that $\alpha=\gamma \Delta$. Let $\gamma_{1}, \gamma_{2} \in I(M)$ satisfy 
$\gamma_{1}(z)=\gamma(z, 0)$ and $\gamma_{2}(z)=\gamma(0, z)(z \in M)$. Then $\alpha=\gamma \Delta=\gamma_{1} \beta_{1}+\gamma_{2} \beta_{2}$, hence $\alpha^{*}=\gamma_{1}^{*} \beta_{1}^{*}+\gamma_{2}^{*} \beta_{2}^{*} \in I(N, y)$. We have shown that $\Phi(I(M, x)) \subseteq$ $I(N, y)$. The reverse inclusion follows from a symmetric argument using $\Delta^{\prime}(N)=\Delta(M)$.

Next we show that there are enough principal elements to generate the module $M$ modulo torsion. Moreover, they can be chosen to satisfy certain conditions. For a torsion element $t$, we let $e(t)$ denote its exponent.

LEMMA 5. Let $M$ be nonsplit with $T(M)$ simply presented. Then

(1) there exist principal elements $x_{i}$ such that $p x_{i+1} \equiv x_{i} \bmod T(M)$ $(i \geq 1)$;

(2) $t_{i}(i \geq 1)$ are independent, where $t_{i}=x_{i}-p x_{i+1}$;

(3) $e\left(t_{i}\right) \leq e\left(p t_{i+1}\right)(i \geq 1)$.

Proof. If the $p$-indicator of $M$ contains infinitely many gaps then by Lemmas 2 and 3 there exist principal elements $u_{i} \in M$ with $p u_{i+1} \equiv u_{i}$ $\bmod T(M)(i \geq 1)$. If the $p$-indictor contains only finitely many gaps then by the nonsplitting of $M$ there exists a $u \in M$ such that $h(u)$ is a limit ordinal $\geq \omega$ and the height sequence of $u$ contains no gaps. Again we invoke the above lemmas to conclude that $u$ is principal. By (4) of Lemma 2 we may choose principal elements $u_{i} \in M$ such that $p^{i} u_{i}=u(i \geq 1)$. Thus we may asume that the $u_{i} \in M$ satisfy (1). Let $x_{1}=u_{1}$. For induction suppose that $x_{1}, x_{2}, \ldots, x_{k}$ have been chosen such that (1), (2), and (3) hold. Let $t=x_{k}-p u_{k+1}$. Since $T(M)$ is unbounded we may choose $s \in T(M)$ such that it is independent of $\left\langle t_{1}, \ldots, t_{k-1}, t\right\rangle$ and $\left\langle p^{e(s)} u_{k+1}\right\rangle=\left\langle u_{k+1}\right\rangle \cap\left\langle x_{k}\right\rangle \cap\left\langle x_{k-1}\right\rangle$. Define $x_{k+1}=u_{k+1}+s$. Clearly $p x_{k+1} \equiv x_{k} \bmod T(M)$. Moreover $\left\langle x_{k-1}\right\rangle \cap\left\langle x_{k}\right\rangle \cap\left\langle u_{k+1}\right\rangle=$ $\left\langle p^{e(s)} u_{k+1}\right\rangle=\left\langle p^{e(s)} x_{k+1}\right\rangle$. By (2) of Lemma 2 it follows that $p^{e(s)} x_{k+1}$ is principal. Another application of (4) of Lemma 2 shows that $x_{k+1}$ is principal and (1) holds. Now $t_{k}=x_{k}-p x_{k+1}=t-p s$. The independence of $\left\{t_{1}, \ldots, t_{k}\right\}$ will follow if $e(p s) \geq e(t)$. But $\left\langle p^{e(s)} u_{k+1}\right\rangle \subseteq\left\langle x_{k}\right\rangle \cap$ $\left\langle u_{k+1}\right\rangle=\left\langle p^{e(t)+1} u_{k+1}\right\rangle$ and therefore $e(s) \geq e(t)+1$. To show (3) we observe that $\left\langle p^{e(s)} u_{k+1}\right\rangle=\left\langle x_{k-1}\right\rangle \cap\left\langle x_{k}\right\rangle \cap\left\langle u_{k+1}\right\rangle=\left\langle p^{e\left(t_{k-1}\right)+1} x_{k}\right\rangle \cap$ $\left\langle u_{k+1}\right\rangle \subseteq\left\langle p^{e\left(t_{k-1}\right)+2} u_{k+1}\right\rangle$ i.e. $e(s) \geq e\left(t_{k-1}\right)+2$. Since $e\left(t_{k}\right) \geq e(p s)$ by the independence of $t$ and $s$ we have $e\left(p t_{k}\right)=e(p s)-1=e(s)-2$ $\geq e\left(t_{k-1}\right)$.

In our final lemma we show that the corresponding principal elements can be chosen to be compatible with $\phi$, the isomorphism on torsion given 
by (A). The latter may require adjustment by a $p$-adic unit. For notational convenience we let $t^{*}$ denote $\phi(t)$ for $t \in T(M)$.

LEMMA 6. Let $\Phi: E(M) \rightarrow E(N)$ be an R-algebra isomorphism, and assume that $M$ is a nonsplit module with $T(M)$ simply presented.

(1) Let $x_{1} \in M, y_{1} \in N$ be principal elements such that $\Phi\left(I\left(M, x_{1}\right)\right)=$ $I\left(N, y_{1}\right)$. If $x_{2} \in M$ is principal with $t=x_{1}-p x_{2}$ torsion, then there exists $a$ principal element $y_{2} \in N$ such that $y_{1}-p y_{2}=k t^{*}, p \nmid k$, and $\Phi\left(I\left(M, x_{2}\right)\right)=I\left(N, y_{2}\right)$.

(2) Let $x_{1}, x_{2}, x_{3} \in M$ with $x_{1}$ principal and let $t_{i}=x_{i}-p x_{i+1}(i=$ $1,2)$ be independent torsion elements with $e\left(t_{1}\right) \leq e\left(p t_{2}\right)$. Suppose further that $y_{l} \in N$ are such that $\Phi\left(I\left(M, x_{i}\right)\right)=I\left(N, y_{i}\right)(i=1,2,3)$ and $y_{i}-$ $p y_{i+1}=k_{i} t_{i}^{*}(i=1,2)$. Then $k_{1} \equiv k_{2} \bmod p^{e\left(t_{1}\right)}$.

Proof. (1) By Lemma 4 there exists a principal element $y_{2} \in N$ such that $\Phi\left(I\left(M, x_{2}\right)\right)=I\left(N, y_{2}\right)$. Let $\left\langle x_{1}\right\rangle \cap\left\langle x_{2}\right\rangle=\left\langle p^{r} x_{1}\right\rangle$. Since $x_{2}$ is principal we conclude that $r$ is minimal such that $I\left(M, x_{2}\right) \subseteq I\left(M, p^{r} x_{1}\right)$. Since $I\left(M, p^{r} x_{1}\right)=\left\{\alpha \in E(M) \mid p^{r} \alpha \in I\left(M, x_{1}\right)\right\}$ and $\Phi\left(I\left(M, x_{1}\right)\right)=$ $I\left(N, y_{1}\right)$, it follows that $\Phi\left(I\left(M, p^{r} x_{1}\right)\right)=I\left(N, p^{r} y_{1}\right)$. Hence $r$ is minimal such that $I\left(N, y_{2}\right) \subseteq I\left(N, p^{r} y_{1}\right)$. This together with $y_{2}$ principal implies that $\left\langle y_{1}\right\rangle \cap\left\langle y_{2}\right\rangle=\left\langle p^{r} y_{1}\right\rangle$. But $t=x_{1}-p x_{2}$, therefore $\left\langle x_{1}\right\rangle \cap\left\langle x_{2}\right\rangle=$ $\left\langle p^{r+1} x_{2}\right\rangle$. By an argument similar to the above and interchanging the roles of $x_{1}, x_{2}$ and $y_{1}, y_{2}$, we obtain $\left\langle y_{1}\right\rangle \cap\left\langle y_{2}\right\rangle=\left\langle p^{r+1} y_{2}\right\rangle$. We may now modify $y_{2}$ by a unit to get $p^{r} y_{1}=p^{r+1} y_{2}$, or $y_{1}-p y_{2}$ torsion. It remains to show that $y_{1}-p y_{2}=k t^{*}, p \nmid k$. From Lemma 2 and the fact that $x_{1}$ is principal it follows that the torsion of the kernel of $I\left(M, x_{1}\right) \cap$ $I\left(M, p x_{2}\right)$ is $\langle t\rangle$. Thus the torsion of the kernel of $I\left(N, y_{1}\right) \cap I\left(N, p y_{2}\right)$ is $\left\langle t^{*}\right\rangle$. Therefore $y_{1}-p y_{2}=k t^{*}$, where $p \nmid k$ since $o\left(y_{1}-p y_{2}\right)=o(t)$.

(2) As above, using the fact that $x_{1}$ is principal we have that the torsion of the kernel of $I\left(M, x_{1}\right) \cap I\left(M, p^{2} x_{3}\right)$ is $\left\langle t_{1}+p t_{2}\right\rangle$. Thus the torsion of the kernel of $I\left(N, y_{1}\right) \cap I\left(N, p^{2} y_{3}\right)$ is $\left\langle t_{1}^{*}+p t_{2}^{*}\right\rangle$. But $y_{1}-$ $p^{2} y_{3}=k_{1} t_{1}^{*}+p k_{2} t_{2}^{*}$, therefore $k_{1} t_{1}^{*}+p k_{2} t_{2}^{*}=l\left(t_{1}^{*}+p t_{2}^{*}\right)$ for some $l$. This implies that $k_{1} \equiv l \bmod p^{e\left(t_{1}\right)}, k_{2} \equiv l \bmod p^{e\left(p t_{2}\right)}$ thus $k_{1} \equiv k_{2} \bmod$ $p^{e\left(t_{1}\right)}$.

We are now ready for the

Proof of the Theorem. We use $\phi$ in (A) to identify the torsion submodules of $M$ and $N$ and denote this common submodule by $T$. By Lemma 1 we may assume $M$ to be nonsplit. Let $\varepsilon$ be an idempotent with 
$\varepsilon(M)=D$, the maximal divisible submodule of torsion, and $(1-\varepsilon)(M)$ $=M_{0}$. Choose $x_{i} \in M(i \geq 1)$ satisfying (1)-(3) of Lemma 5. By Lemmas 4 and 6 we choose principal $y_{i} \in N$ inductively such that

(a) $\Phi\left(I\left(M, x_{i}\right)\right)=I\left(N, y_{i}\right)(i \geq 1)$,

(b) $y_{i}-p y_{i+1}=k_{i} t_{i}^{*}, p \nmid k_{i}(i \geq 1)$,

(c) $k_{i} \equiv k_{i+1} \bmod p^{e\left(t_{i}\right)}(i \geq 1)$.

Since $\left\{e\left(t_{i}\right)\right\}$ is monotonic there exists a unit $\eta$ in the $p$-adic completion of $R$ such that $\eta t_{i}^{*}=k_{i} t_{i}^{*}(i \geq 1)$. Define $R$-isomorphisms $\theta_{i}: T \oplus\left\langle x_{i}\right\rangle \rightarrow T$ $\oplus\left\langle y_{i}\right\rangle$ by $\left.\theta_{i}\right|_{T}=\eta \phi$ and $\theta_{i}\left(x_{i}\right)=y_{i}$. We claim that $\left.\theta_{i+1}\right|_{T \oplus\left\langle x_{i}\right\rangle}=\theta_{i}$. It suffices to show that $\theta_{i+1}\left(x_{i}\right)=\theta_{i}\left(x_{i}\right)$. However

$$
\begin{aligned}
\theta_{i+1}\left(x_{i}\right) & =\theta_{i+1}\left(p x_{i+1}+t_{i}\right)=p y_{i+1}+\eta t_{i}^{*} \\
& =p y_{i+1}+k_{i} t_{i}^{*}=y_{i}=\theta_{i}\left(x_{i}\right) .
\end{aligned}
$$

Put $\theta=\cup_{i} \theta_{i}$. Since $\cup_{i}\left(T \oplus\left\langle x_{i}\right\rangle\right)=M$ and $\cup_{i}\left(T \oplus\left\langle y_{i}\right\rangle\right)=N, \theta$ is an $R$-isomorphism from $M$ to $N$. We note for later use that for $\alpha \in E(M)$, $\left.\Phi(\alpha)\right|_{T}=\left.\phi \alpha \phi^{-1}\right|_{T}=\left.\theta \alpha \theta^{-1}\right|_{T}$.

We now show that $E(M)=R+I(M)$. This will hold if we can prove that no $\alpha \in E(M)$ induces multiplication by $1 / p$ on $M / T$. If such an $\alpha$ exists, then for torsion-free $x \in M, p \alpha(x)=x+t$ for some $t \in T$. Thus $\alpha\left(p^{n+1} x\right)=p^{n} x$ for some $n$, whence $h\left(p^{n} x\right)=h\left(\alpha\left(p^{n+1} x\right)\right) \geq h\left(p^{n+1} x\right)$ $>h\left(p^{n} x\right)$, a contradiction. In view of this we need to show $\alpha^{*}=\theta \alpha \theta^{-1}$ only for $\alpha \in I(M)$. In fact if we put $T\left(M_{0}\right)=T_{0}$ then it suffices to consider $\alpha$ such that either $\alpha\left(M_{0}\right) \subseteq T_{0}$ or $\alpha\left(M_{0}\right) \subseteq D$. Before we treat these cases we prove a claim: $\varepsilon^{*}=\boldsymbol{\theta} \varepsilon \theta^{-1}$. Since $\theta \varepsilon \theta^{-1}$ is an idempotent yielding the decomposition $\theta\left(M_{0}\right) \oplus D$ it suffices to show that $\varepsilon^{*}$ yields this decomposition also. Clearly $\left.\varepsilon^{*}\right|_{D}=1_{D}$. Note that $M_{0}=\left\langle\left\{x_{i}\right\}, T_{0}\right\rangle$ and therefore $\theta\left(M_{0}\right)=\left\langle\left\{y_{i}\right\}, T_{0}\right\rangle$. Since $\varepsilon^{*}\left(T_{0}\right)=\varepsilon\left(T_{0}\right)=0$ and from (a) $\varepsilon^{*} \in I\left(N, y_{i}\right)(i \geq 1)$ we have $\varepsilon^{*} \theta\left(M_{0}\right)=0$. Thus our claim is shown and we return to our two cases.

First suppose that $\alpha\left(M_{0}\right) \subseteq T_{0}$. This is equivalent to $\alpha \varepsilon=\varepsilon \alpha$. Therefore $\alpha^{*} \varepsilon^{*}=\varepsilon^{*} \alpha^{*}$ and by part (1) of the proof of Lemma 4 it follows that $\alpha^{*}\left(N_{0}\right) \subseteq T_{0}$ where $N_{0}=\left(1-\varepsilon^{*}\right)(N)$. Now $\theta \alpha \theta^{-1} \varepsilon^{*}=\theta \alpha \varepsilon \theta^{-1}=\theta \varepsilon \alpha \theta^{-1}$ $=\varepsilon^{*} \theta \alpha \theta^{-1}$ and hence $\theta \alpha \theta^{-1}\left(N_{0}\right) \subseteq T_{0}$. However this together with $\alpha^{*}\left(N_{0}\right)$ $\subseteq T_{0},\left.\alpha^{*}\right|_{T}=\left.\theta \alpha \theta^{-1}\right|_{T}$, and $\operatorname{Hom}\left(N / T, T_{0}\right)=0$ allows us to conclude that $\alpha^{*}=\theta \alpha \theta^{-1}$. Now we suppose $\alpha\left(M_{0}\right) \subseteq D$. Let $x \in M$ be principal. Then $p^{r} \alpha(x)=0$ for some $r$. By an argument in part (2) of the proof of Lemma $4, p^{r} \alpha=\gamma_{1} \beta_{1}+\gamma_{2} \beta_{2}$ where $\gamma_{i}\left(M_{0}\right) \subseteq D$ and $\beta_{i}\left(M_{0}\right) \subseteq T_{0}(i=$ 1,2).By the previous case and the fact that $\gamma_{l}$ in the above equation are applied only to torsion elements, we have $p^{r} \alpha^{*}=\gamma_{1}^{*} \beta_{1}^{*}+\gamma_{2}^{*} \beta_{2}^{*}=$ $\theta^{-1}\left(\gamma_{1} \beta_{1}+\gamma_{2} \beta_{2}\right) \theta=p^{r} \theta \alpha \theta^{-1}$. Now $\varepsilon \alpha=\alpha$ implies $\varepsilon^{*} \alpha^{*}=\alpha^{*}$ and therefore $\alpha^{*}(N) \subseteq D$. Clearly $\theta \alpha \theta^{-1}(N) \subseteq D$. Since $\left.\alpha^{*}\right|_{T}=\left.\theta \alpha \theta^{-1}\right|_{T}$ and 
$\operatorname{Hom}(N / T, D)$ is torsion-free we have $\alpha^{*}=\theta \alpha \theta^{-1}$ to complete the proof.

We close the paper with two questions. Does the theorem hold for arbitrary torsion submodules? Here, different methods may be required since our proof relies to a large degree on the result in [4] proved for simply presented torsion. A key step would be to reprove Lemma 4 in this new setting. The second question concerns higher rank. Does the theorem hold for modules of countable torsion-free rank over complete discrete valuation rings? The ring needs to be complete to eliminate pathological examples of torsion-free modules of finite rank that occur over incomplete discrete valuation rings. In addition the rank must be countable in view of an example in [3, §4].

\section{REFERENCES}

1. L. Fuchs, Infinite Abelian Groups, Vol. II, Academic Press, New York, 1973.

2. I. Kaplansky, Infinite Abelian Groups, University of Michigan Press, Ann Arbor, 1969.

3. W. May and E. Toubassi, Endomorphisms of abelian groups and the theorem of Baer and Kaplansky, J. Algebra, 43 (1976), 1-13.

4. ___ Embedding of totally projective groups, Arch. Math., 29 (1977), 465-471.

$5 . \quad$, Isomorphisms of endomorphism rings of rank one mixed groups, J. Algebra, 71 (1981), 508-514.

6. K. Wallace, On mixed groups of torsion-free rank one with totally projective primary components, J. Algebra, 17 (1971), 482-488.

Received November 2, 1981.

UNIVERSITY OF ARIZONA

TUCSON, AZ 85721 



\section{PACIFIC JOURNAL OF MATHEMATICS EDITORS}

Donald BABBITT (Managing Editor)

University of California

Los Angeles, CA 90024

Hugo Rossi

University of Utah

Salt Lake City, UT 84112

C. C. Moore and Arthur Ogus

University of California

Berkeley, CA 94720
J. DugundiI

Department of Mathematics

University of Southern California

Los Angeles, CA 90089-1113

R. Finn and H. SAMELSON

Stanford University

Stanford, CA 94305

ASSOCIATE EDITORS
R. ARENS
E. F. BECKENBACH
B. H. NeUmanN
F. WOLF
K. YosHIDA (1906-1982)

\section{SUPPORTING INSTITUTIONS}

UNIVERSITY OF ARIZONA

UNIVERSITY OF BRITISH COLUMBIA

CALIFORNIA INSTITUTE OF TECHNOLOGY

UNIVERSITY OF CALIFORNIA

MONTANA STATE UNIVERSITY

UNIVERSITY OF NEVADA, RENO

NEW MEXICO STATE UNIVERSITY

OREGON STATE UNIVERSITY
UNIVERSITY OF OREGON

UNIVERSITY OF SOUTHERN CALIFORNIA

STANFORD UNIVERSITY

UNIVERSITY OF HAWAII

UNIVERSITY OF TOKYO

UNIVERSITY OF UTAH

WASHINGTON STATE UNIVERSITY

UNIVERSITY OF WASHINGTON 


\section{Pacific Journal of Mathematics}

\section{Vol. 108, No. $1 \quad$ March, 1983}

Waleed A. Al-Salam and A. Verma, $q$-Konhauser polynomials $\ldots \ldots \ldots \ldots 1$

Alfred David Andrew, The Banach space JT is primary $\ldots \ldots \ldots \ldots \ldots . . .6$

Thomas E. Bengtson, Bessel functions on $P_{n} \ldots \ldots \ldots \ldots \ldots \ldots$

Joaquim Bruna Floris and Francesc Tugores, Free interpolation for

holomorphic functions regular to the boundary $\ldots \ldots \ldots \ldots \ldots \ldots \ldots \ldots$

Peter Dierolf and Susanne Dierolf, Topological properties of the dual pair

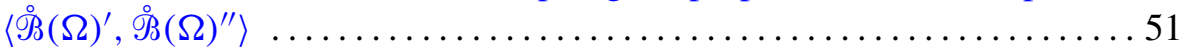

Gerald Arthur Edgar, An ordering for the Banach spaces $\ldots \ldots \ldots \ldots \ldots 83$

Basil Gordon, A proof of the Bender-Knuth conjecture . . . . . . . . . . . . . 99

Harold T. Hodes, A minimal upper bound on a sequence of Turing degrees

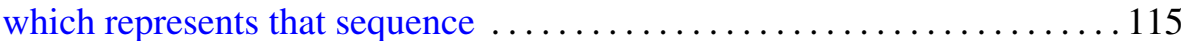

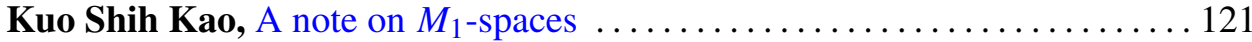

Frank Kost, Topological extensions of product spaces ................ 129

Eva Lowen-Colebunders, On the convergence of closed and compact

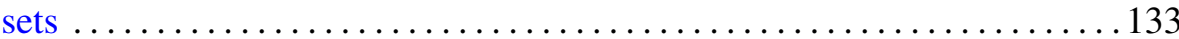

Doron Lubinsky, Divergence of complex rational approximations . . . . . . 141

Warren May and Elias Hanna Toubassi, Endomorphisms of rank one

mixed modules over discrete valuation rings $\ldots \ldots \ldots \ldots \ldots \ldots \ldots \ldots \ldots$

Richard Patrick Morton, The quadratic number fields with cyclic

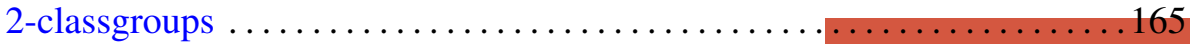

Roderic Murufas, Rank of positive matrix measures . . . . . . . . . . . . 177

Helga Schirmer, Fixed point sets of homotopies . . . . . . . . . . . . . 191

E. Taflin, Analytic linearization of the Korteweg-de Vries equation ........ 203

James Thomas Vance, Jr., $L^{p}$-boundedness of the multiple Hilbert

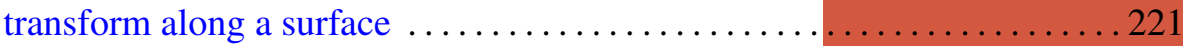

Hiroshi Yamaguchi, A property of some Fourier-Stieltjes transforms . . . . . 243 